\title{
Ecology: Functions, Patterns, and Evolution
}

\author{
Fernando Valladares \\ Centro de Ciencias Medioambientales, Madrid, Spain
}

\section{INTRODUCTION}

Ecology is the study of living organisms and their environment in an attempt to explain and predict. While natural history involves the accumulation of detailed data with emphasis on the autecology of each species, the objectives of ecology in general and of functional ecology in particular are to develop predictive theories and to assemble the data to develop general models. Functional ecology has three basic components: 1) constructing trait matrices through screening of various plant and animal species, 2) exploring empirical relationships among these traits, and 3) determining the relationships between traits and environments. ${ }^{[1]}$ Studies in functional ecology encompass a wide range of approaches, from individuals to populations; from mechanistically detailed to deliberately simplified, black-box simulations; and from deductive to inductive. ${ }^{[2]}$ Functional ecology is concerned with the links between structure and function, the existence of general patterns among species, and the evolutionary connections among these patterns. And functional ecology is, above all, timely and pertinent, because the environmental degradation associated with human development is rapidly destroying the very systems that ecologists seek to understand. If we are to anticipate the extent and repercussions of global change in natural habitats, we first need to understand how organisms and ecosystems function.

\section{EXPLORING THE MECHANISMS: FROM PHYSIOLOGY TO ECOPHYSIOLOGY AND FUNCTIONAL ECOLOGY}

The study of plant functions has largely followed a reductionistic approach aimed at explaining the functions in terms of the principles of physics and chemistry. However, plant physiology, which is focused on molecules, organelles, and cells, has not been able to provide reliable predictions of the responses of vegetation to changes in their environment, due to the multiple interactions involved in the responses of plants and the hierarchical nature of plant organization. ${ }^{[3]}$ The realization of the fact that the responses of higher organizational levels are not predictable from the dynamics of those of smaller scales led to the advent of plant ecophysiology, which is focused on organs-a more relevant scale of organization to address questions regarding plant performance. Ecophysiologists have primarily focused on the structural and functional properties of leaves. Leaves display wide variation in morphology and physiology, including differences in specific mass, carbon and nitrogen investments, stomatal densities, optical properties, and hydraulic and photosynthetic characteristics. The emphasis of ecophysiological studies on leaves is due to the profound implications of the interactions between leaf structure and function for the performance of plants in natural habitats. ${ }^{[4,5]}$ However, the traditional ecophysiological approach proved to be insufficient in predicting plant distribution and responses to changing environments, which led to the development of functional plant ecology. ${ }^{[6]}$ Functional ecology is centered on whole plants as the unit of analysis, encompassing a range of scales of organization from organs to whole organism architecture, and is based on a much broader conception of plant functions than that formulated by the earlier practitioners of ecophysiology. ${ }^{[7]}$

\section{THE SEARCH OF GENERAL PATTERNS: COMPARATIVE ECOLOGY}

Since elucidation of the range of possible functional responses of plants is not possible with the use of model organisms, such as those typically used in plant physiology, functional ecology arises as an essentially comparative science. Ideally, functional ecology deals with traits measured on a large number of species in order to minimize the influence of the peculiarities of the autecology of each species. Two main approaches have been followed to find general ecological patterns in nature: 1) screening, that is, the design of bioassays for a trait or a set of traits measured simultaneously on a large number of species, as in the classic study by Grime and Hunt $^{[8]}$ of the relative growth rate of 132 species of British flora, and 2) empiricism, or the search for quantitative relationships between measurable dependent and independent variables (e.g., correlations among pairs 
of traits or traits and environments) producing quantitative models using traits and not species, as in the general revision of leaf traits by Reich et al. ${ }^{[4]}$ Models using traits are more general than those based on species and can be more easily transferred to different floras. ${ }^{[1]}$ The question arises as to which trait must be measured. A possible answer can be obtained by analyzing the basic functions that organisms perform (i.e., resource acquisition, the ability to tolerate environmental extremes, and the ability to compete with neighbors) and then either looking for traits which measure these functions or carrying out direct bioassays of them. The most common limitations of this kind of study are the difficulties in finding unambiguous linkages of a trait to a specific function, and the so-called phylogenetic constraints that are due to the fact that phylogenetic proximity among species can influence their functional similarities.

Individual plants demonstrate important degrees of phenotypic variation, which must be considered in comparative studies. No two individuals of the same species exhibit the same final shape or functional features, regardless of how similar the genotypes of two individuals may be. ${ }^{[9]}$ Part of this variation is due to phenotypic plasticity, that is, the capacity of a given genotype to render different phenotypes under different environmental conditions, and part is due to other reasons (ontogenetic stage, developmental instability). Plants of the same chronological age can be ontogenetically different, so interpretation of differences in phenotypic traits will depend on whether comparisons are made as a function of age, size, or developmental stage.

\section{EXPERIMENTAL ECOLOGY}

Broad-scale comparisons must be driven by hypotheses and must be based on robust statistical designs. However, the finding of statistically significant patterns is no guarantee of underlying cause-and-effect relationships, which must be tested experimentally. Gradient analysis, where functional responses are examined along a clearly defined environmental gradient, is a powerful approach to exploring the relationships between plant function and environment, but it is prone to spurious relationships when there is a hidden factor covarying with the factor defining the gradient. ${ }^{[3]}$ Inferences from gradient analysis and broad-scale comparisons are statistical in nature and must be confirmed experimentally.

Experiments are designed to test hypotheses, but sufficient knowledge must be available to specify more than a trivial hypothesis before thorough experimentation can be undertaken. ${ }^{[2]}$ Unfortunately, this is not the case for many natural systems. Although experiments are a common practice in physiology and ecophysiology, where mechanisms can be explored and hypotheses tested under relatively well-controlled conditions, they are less common in functional ecology studies, especially when hypotheses must be tested in natural habitats. Experimental design should meet a number of standards that are not easy - and in certain cases not possible - to meet in field ecology. The controls should be randomly intermixed with the treatments, both in space and time, and both the control and the experiments must be replicated enough. Replicates are not easy to find in natural scenarios, and when they can be found they are frequently not truly independent of one another, leading to a weak experimental design due to pseudoreplication. In addition, multiple causality and indirect effects, which significantly complicate the interpretation of results, are commonplace in ecology. However, experimental ecology is burgeoning despite all this adversity, because experiments are irreplaceable elements in achieving relevant progress in our understanding of ecological processes, as has been revealed by a number of experimental manipulations of natural populations. ${ }^{[10]}$

\section{EVOLUTIONARY ECOLOGY}

Functional ecology eventually leads to evolutionary ecology. Trends in functional traits across species and mechanisms linking cause and effect contribute to our understanding of evolutionary processes, especially when they are considered on a time scale long enough to allow for changes in gene frequencies, the essence of evolution. Whereas functional ecology is interested in the immediate influence of environment on a given trait, evolutionary ecology is aimed at understanding why some individuals have left the most offspring in response to long-term consistent patterns of environmental conditions. Functional ecology, focused on an ecological time scale (now time), asks questions of "how?' and is concerned with the proximate factors influencing an event. Evolutionary ecology is focused on an evolutionary time scale (geological time), asking questions of "why?', and concerned with the ultimate factors influencing an event. Neither is more correct than the other, and they are not mutually exclusive because ecological events can always be profitably considered within an evolutionary framework, and vice versa. ${ }^{[10]}$

There are five agents of evolution: natural selection (differential reproductive success of individuals within a population); genetic drift (random sampling bias in small populations); gene flow (migration movements of individuals among and between populations with different gene frequencies); meiotic drive (segregation

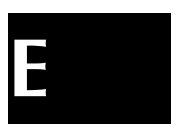


distortion of certain alleles that do not follow the Mendelian lottery of meiosis and recombination); and mutation. Of these agents, only natural selection is directed, resulting in conformity between organisms and their environments. ${ }^{[10]}$ Darwin's theory of natural selection is a fundamental unifying theory, and the many studies that have been carried out over the last century in support of it demonstrate the power of the rigorous application of the genetic theory of natural selection to population biology.

\section{ARTICLES OF FURTHER INTEREST}

Breeding: Genotype-by-Environment Interaction, p. 218

Coevolution of Insects and Plants, p. 289

Competition: Responses to Shade by Neighbors, p. 300

Cross-species and Cross-genera Comparisons in the Grasses, p. 374

Ecology and Agricultural Sciences, p. 401

Ecophysiology, p. 410

Photosynthesis and Stress, p. 901

Plant Response to Stress: Mechanisms of Accommodation, p. 987

Population Genetics, p. 1042

Shade Avoidance Syndrome and Its Impact on Agriculture, p. 1152

\section{REFERENCES}

1. Keddy, P.A. A pragmatic approach to functional ecology. Funct. Ecol. 1992, 6, 621-626.

2. Mentis, M.T. Hypothetico-deductive and inductive approaches in ecology. Funct. Ecol. 1988, 2, 5-14.

3. Duarte, C. Methods in Comparative Functional Ecology. In Handbook of Functional Plant Ecology; Pugnaire, F.I., Valladares, F., Eds.; Marcel Dekker: New York, 1999; 1-8.

4. Reich, P.B.; Ellsworth, D.S.; Walters, M.B.; Vose, J.M.; Gresham, C.; Volin, J.C.; Bowman, W.D. Generality of leaf trait relationships: A test across six biomes. Ecology 1999, 80, 1955-1969.

5. Niinemets, U. Global-scale climatic controls of leaf dry mass per area, density, and thickness in trees and shrubs. Ecology 2001, 82, 453-469.

6. Pugnaire, F.I.; Valladares, F. Handbook of Functional Plant Ecology; Marcel Dekker: New York, 1999.

7. Valladares, F.; Pearcy, R.W. The functional ecology of shoot architecture in sun and shade plants of Heteromeles arbutifolia M. Roem., a Californian chaparral shrub. Oecologia 1998, 114, 1-10.

8. Grime, J.P.; Hunt, R. Relative growth rate: Its range and adaptive significance in a local flora. J. Ecol. 1975, 63, $393-422$.

9. Coleman, J.S.; McConnaughay; Ackerley, D.D. Interpreting phenotypic variation in plants. Trends Ecol. Evol. 1994, 9, 187-191.

10. Pianka, E.R. Evolutionary Ecology; Benjamin-Cummings: San Francisco, 2000. 


\section{Request Permission or Order Reprints Instantly!}

Interested in copying and sharing this article? In most cases, U.S. Copyright Law requires that you get permission from the article's rightsholder before using copyrighted content.

All information and materials found in this article, including but not limited to text, trademarks, patents, logos, graphics and images (the "Materials"), are the copyrighted works and other forms of intellectual property of Marcel Dekker, Inc., or its licensors. All rights not expressly granted are reserved.

Get permission to lawfully reproduce and distribute the Materials or order reprints quickly and painlessly. Simply click on the "Request Permission/ Order Reprints" link below and follow the instructions. Visit the U.S. Copyright Office for information on Fair Use limitations of U.S. copyright law. Please refer to The Association of American Publishers' (AAP) website for guidelines on Fair Use in the Classroom.

The Materials are for your personal use only and cannot be reformatted, reposted, resold or distributed by electronic means or otherwise without permission from Marcel Dekker, Inc. Marcel Dekker, Inc. grants you the limited right to display the Materials only on your personal computer or personal wireless device, and to copy and download single copies of such Materials provided that any copyright, trademark or other notice appearing on such Materials is also retained by, displayed, copied or downloaded as part of the Materials and is not removed or obscured, and provided you do not edit, modify, alter or enhance the Materials. Please refer to our Website User Agreement for more details.

\section{Request Permission/Order Reprints}

Reprints of this article can also be ordered at http://www.dekker.com/servlet/product/DOI/101081EEPCS120010498 